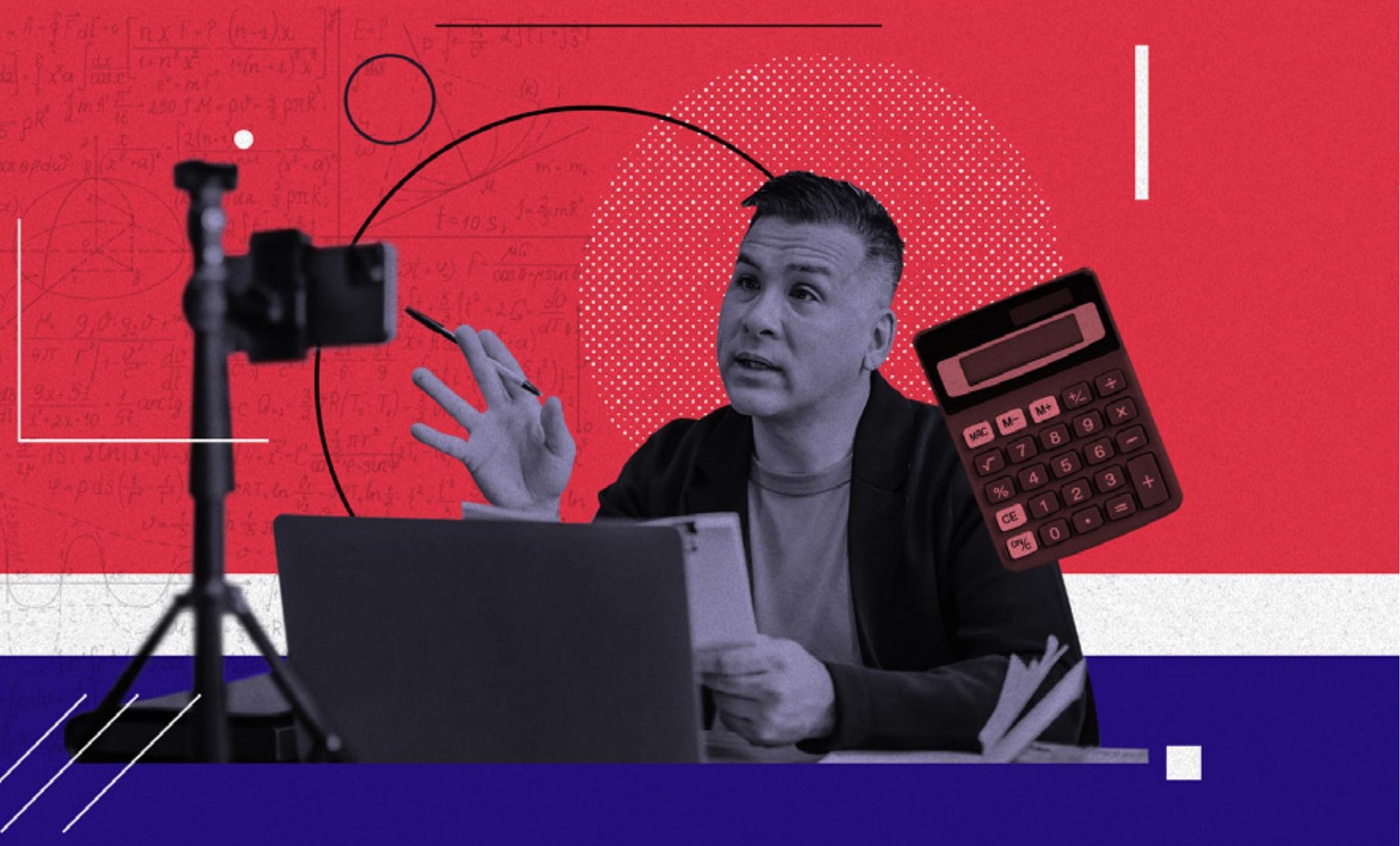

\title{
El impacto de un curso virtual propedéutico en el aprendizaje de las matemáticas para aspirantes a nivel medio superior en tiempos de pandemia
}

THE IMPACT OF A VIRTUAL PREPARATORY COURSE IN MATHEMATICS LEARNING FOR ASPIRING HIGH SCHOOL LEVEL IN TIMES OF PANDEMIC

O IMPACTO DE UM CURSO VIRTUAL PREPARATÓRIO NA APRENDIZAGEM DE MATEMÁTICA PARA CANDIDATOS AO ENSINO MÉDIO EM TEMPOS DE PANDEMIA

José Eduardo Rodríguez Guevaraㅁ; Luis Alberto Soto Reyes ${ }^{2}$ 


\section{Citar artículo como:}

Rodríguez-Guevara, J.E., y Soto-Reyes, L.A. (2021). El impacto de un curso virtual propedéutico en el aprendizaje de las matemáticas para aspirantes a nivel medio superior en tiempos de pandemia. Educación y Ciudad, n. 41, pp. 133- 145. https://doi.org/10.36737/01230425.n41.2021.2511

Fecha de recepción: 29 de marzo de 2021

Fecha de aprobación: 6 de julio de 2021

\section{Resumen}

La Escuela de Bachilleres de la Universidad Autónoma de Querétaro (EBUAQ) como parte de su proceso de admisión, oferta su curso propedéutico con el objetivo de fortalecer los aprendizajes que serán evaluados en el EXCOBA, prueba estandarizada que define el ingreso de los aspirantes. Este curso pasó a la virtualidad debido al gran número de aspirantes, y para resguardar su salud en tiempos de pandemia, en la presente propuesta se describe el impacto que tuvo en el estudio de las matemáticas.

Palabras clave: Matemáticas; propedéutico; entorno virtual de aprendizaje; EXCOBA.

\section{Abstract}

The High School of the Autonomous University of Querétaro (EBUAQ) as part of their admissions process, offers its preparatory course in order to strengthen the learning that will be evaluated in the EXCOBA, standardized test that defines the entrance income of applicants. This course passed to virtuality due to the large number of applicants and to protect their health in times of pandemic. This proposal describes the impact it had on the study of mathematics.

Keywords: Mathematics; Propaedeutic; Virtual Learning Environment; EXCOBA.

\section{Resumo}

Como parte de seu processo seletivo, a Escola de Bacharelado da Universidade Autônoma de Querétaro (EBUAQ) oferece seu curso preparatório com o objetivo de fortalecer a aprendizagem que será avaliada no EXCOBA, uma prova padronizada que define a admissão dos candidatos. O referido curso foi migrado para a virtualidade devido ao grande número de candidatos e para proteger sua saúde em tempos de pandemia, esta proposta descreve o impacto que teve no estudo da matemática.

Palavras-chave: Matemática; Propedêutico; Ambiente Virtual de Aprendizagem e EXCOBA. 


\section{Introducción}

L as matemáticas son una de las áreas donde los estudiantes enfrentan una mayor dificultad a la hora de lograr asimilar conocimientos significativos, de hecho, en México más del $50 \%$ de los alumnos de educación básica no alcanzan el nivel mínimo de competencias en este en esta área (Organización para la Cooperación y el Desarrollo Económicos, 2018). Estos problemas en el aprendizaje de las matemáticas son multifactoriales, sin embargo, algunos de los más significativos son la falta de preparación por parte de los docentes y el incorrecto aprendizaje de contenidos previos que son requeridos para el estudio del álgebra (Cuevas, 2014).

Sumado a esto, la mayoría de las instituciones educativas tuvieron que afrontar una migración de cursos presenciales a una modalidad virtual, debido a circunstancias extraordinarias que restringen las metodologías presenciales, y que nos ha hecho migrar a una modalidad virtual. Lo anterior, como parte de las medidas preventivas impuestas por los gobiernos y la Organización Mundial de la Salud (Secretaría de Salud, 2020). Por este motivo, fue importante comprender que este cambio implicaba generar tecnología educativa, lo cual significa articular recursos técnicos y humanos que deben interactuar para concebir, aplicar y evaluar procesos de enseñanza-aprendizaje (Cabero, 2015).

En este contexto, la Escuela de Bachilleres de la Universidad Autónoma de Querétaro (EBUAQ), ofertó

\footnotetext{
Universidad Autónoma de Querétaro. Maestro en Innovación de Entornos Virtuales de Enseñanza-Aprendizaje. ORCID: https://orcid.org/0000-0002-4402-5598 Correo Electrónico: jose.eduardo.rodriguez@uaq.mx
}

un curso virtual propedéutico a sus aspirantes, cuyo objetivo es fortalecer su conocimiento en aquellos temas que serán evaluados en la prueba estandarizada EXCOBA, la cual define su ingreso a la institución. Los contenidos de este curso se alojaron en la tecnología de Moodle que se define como un Learning Managment System (LMS), es decir, una plataforma a través de la cual se pueden gestionar el aprendizaje mediante la distribución de recursos y actividades para el estudiante (Caletti y Romero, 2015).

\section{Metodología}

El diseño de los materiales de estudio exigió evitar prácticas que orientarán a los alumnos a un aprendizaje memorístico, promoviendo el desarrollo del autoaprendizaje, el análisis de casos de estudio y la interpretación de contenidos didácticos. De esa manera el diseño de los recursos tenía una estructura homogenizada definida de la siguiente manera:

- Presentación teórica que oriente a la reflexión

- Ejemplos que favorezcan el análisis

- Actividades que generen un aprendizaje significativo

Este proceso de diseño de contenido se basó en la comprensión de su naturaleza y en cómo asimilan los estudiantes la nueva información. De manera general, se tomó en cuenta la siguiente secuencia para diseñar los contenidos:

Universidad Autónoma de Querétaro. Maestro en Ciencias en Ingeniería Matemáticas. ORCID: https://orcid.org/0000-0002-6074-375X Correo electrónico: luis.soto@ uaq. $\mathrm{mx}$ 
- Visión general: se presenta material nuevo y se activan los conocimientos previos para iniciar una interacción.

- Asimilación: Se provocan situaciones de aprendizaje en las que el alumno tenga la oportunidad de establecer la relación entre el material nuevo y el material ya conocido.

- Sistematización: Se diseñan situaciones de aprendizaje encaminadas a provocar el cambio cognitivo por medio de la integración de la información anterior con la nueva.

- Aplicación: El nuevo conocimiento se aplica a una situación o ejemplo para resolver un problema real o verosímil.

En la presente propuesta se describe de manera general los resultados que obtuvieron los estudiantes que tomaron el curso propedéutico virtual de la Escuela de Bachilleres de la Universidad Autónoma de Querétaro (EBUAQ). Inicialmente, se hace referencia a los resultados obtenidos en el examen de matemáticas del curso propedéutico y posteriormente se describe el impacto de los diferentes recursos didácticos incluidos en este curso como parte de una estrategia de enseñanza en términos de la funcionalidad del curso.

Cabe mencionar que durante el desarrollo del proyecto se trabajó con dos grupos de alumnos, uno de ellos conformado por estudiantes del curso propedéutico de la EBUAQ al cual nos referiremos como grupo experimental, y el otro, llamado grupo de control, integrado por aspirantes que no cursaron el curso propedéutico. El primer grupo presentó dos exámenes, uno virtual y otro presencial, mientras que el otro grupo sólo presentó la prueba presencial. Los resultados obtenidos por los estudiantes en dichas evaluaciones se analizaron cuantitativamente
(Hernández et al., 2010,) con el objetivo de identificar sus fortalezas y debilidades de aprendizaje.

\section{Resultados}

Los temas incluidos en los contenidos del curso propedéutico de la EBUAQ para reafirmar el conocimiento sobre matemáticas de los estudiantes fueron:

- Números, operaciones y figuras

- Magnitudes, proporciones, probabilidad y estadística

- Álgebra

- Geometría

- Estadística

Al final se realizó una evaluación mediante un examen virtual que incluía 35 preguntas sobre los distintos temas. Además, los subtemas que se evaluaron en cada tema fueron los mismos que se consideran en el diseño de la prueba estandarizada EXCOBA, la cual deben presentar los aspirantes de la EBUAQ como parte de su proceso de admisión.

En la Figura 1, se observa el porcentaje de aciertos que tuvieron en cada tema, el mayor porcentaje obtenido por los estudiantes se vincula con preguntas sobre magnitudes, proporciones, probabilidad y estadística $(68,28 \%)$, mientras que el menor porcentaje con reactivos sobre estadística $(47,14 \%)$. Aunque en ambos temas se incluye el estudio de la estadística, en el primero de éstos se centró la atención en la noción de esta rama de las matemáticas, el cálculo de medidas de tendencia central y la organización y representación de datos en una gráfica de barras, mientras que en el otro se estudió desde su definición hasta el cálculo del rango, la desviación estándar y varianza. Esto se puede relacionar con 
varios aspectos, aún cuando los sujetos de la muestra ya habían estudiado diversos contenidos sobre estadística de acuerdo con lo que se establece en los planes y programas de estudio (Secretaría de Educación Pública, 2015, 2017, 2018) durante los tres años que cursaron la escuela secundaria.

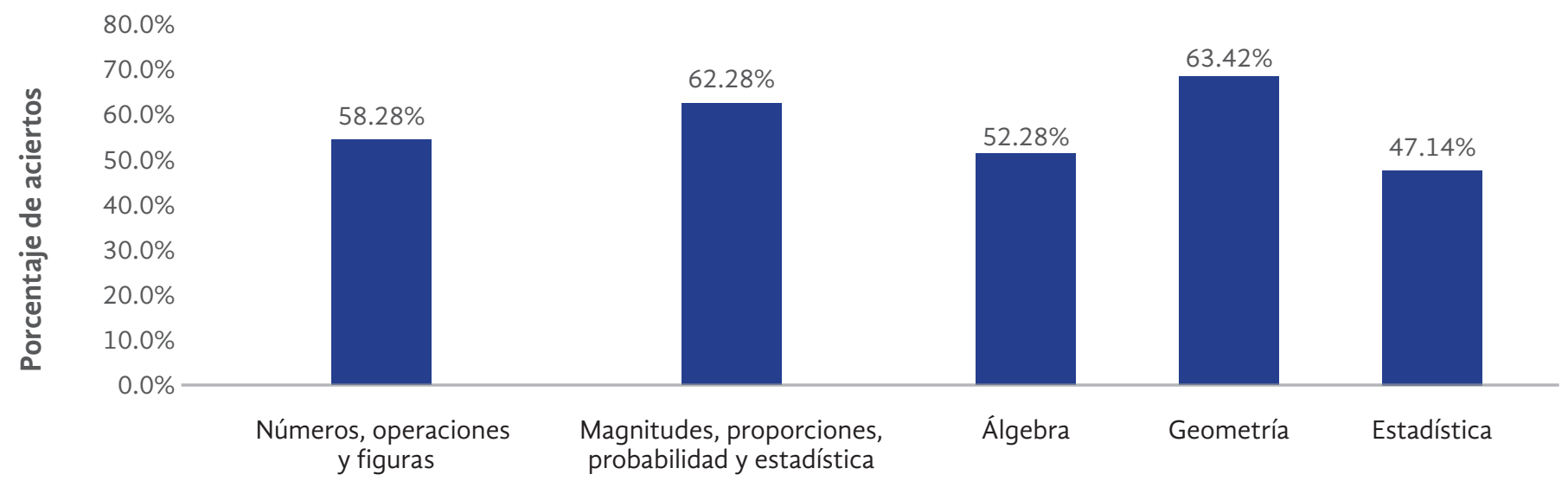

Figura 1. Porcentajes de aciertos en los diferentes temas del examen de matemáticas del curso propedéutico de la EBUAQ.

Fuente: Elaboración propia.

Los aciertos obtenidos en cada uno de los reactivos de los subtemas del examen de matemáticas del curso propedéutico de la EBUAQ se pueden asociar con las fortalezas y debilidades de los estudiantes tal y como se puede observar en la Tabla 1.

Tabla 1. Preguntas por subtema y número de aciertos en cada una de éstas.

\begin{tabular}{|c|c|c|c|c|c|}
\hline Temas & Subtemas & $\begin{array}{c}\text { Cantidad } \\
\text { de preguntas }\end{array}$ & $\begin{array}{c}\mathrm{N}^{\circ} \text { de } \\
\text { Pregunta }\end{array}$ & Aciertos & $\%$ Tema \\
\hline \multirow{7}{*}{$\begin{array}{l}\text { Números, } \\
\text { operaciones y } \\
\text { figuras }\end{array}$} & \multirow{2}{*}{$\begin{array}{l}\text { Números naturales y } \\
\text { decimales }\end{array}$} & \multirow{2}{*}{2} & 1 & 34 & \multirow{7}{*}{58,28} \\
\hline & & & 2 & 23 & \\
\hline & \multirow{2}{*}{ Números fraccionarios } & \multirow{2}{*}{2} & 3 & 22 & \\
\hline & & & 4 & 30 & \\
\hline & \multirow{2}{*}{ Figuras planas } & \multirow{2}{*}{2} & 5 & 28 & \\
\hline & & & 6 & 37 & \\
\hline & Cuerpos geométricos & 1 & 7 & 30 & \\
\hline
\end{tabular}

Continúa 
Continuación Tabla 1. Preguntas por subtema y número de aciertos en cada una de éstas.

\begin{tabular}{|c|c|c|c|c|c|}
\hline Temas & Subtemas & $\begin{array}{c}\text { Cantidad } \\
\text { de preguntas }\end{array}$ & $\begin{array}{c}\mathrm{N}^{\circ} \text { de } \\
\text { Pregunta }\end{array}$ & Aciertos & \% Tema \\
\hline \multirow{7}{*}{$\begin{array}{l}\text { Magnitudes, } \\
\text { proporciones, } \\
\text { probabilidad y } \\
\text { estadística }\end{array}$} & \multirow{3}{*}{ Magnitudes } & \multirow{3}{*}{3} & 8 & 29 & \multirow{7}{*}{68,28} \\
\hline & & & 9 & 36 & \\
\hline & & & 10 & 40 & \\
\hline & \multirow{2}{*}{$\begin{array}{l}\text { Relaciones de } \\
\text { proporcionalidad }\end{array}$} & \multirow{2}{*}{2} & 11 & 35 & \\
\hline & & & 12 & 30 & \\
\hline & $\begin{array}{l}\text { Nociones de } \\
\text { probabilidad }\end{array}$ & 1 & 13 & 35 & \\
\hline & Nociones de estadística & 1 & 14 & 34 & \\
\hline \multirow{7}{*}{ Álgebra } & \multirow{2}{*}{ Números reales } & \multirow{2}{*}{2} & 15 & 23 & \multirow{7}{*}{52,28} \\
\hline & & & 16 & 27 & \\
\hline & Sucesiones aritméticas & 1 & 17 & 30 & \\
\hline & Polinomios & 1 & 18 & 25 & \\
\hline & Ecuación lineal & 1 & 19 & 31 & \\
\hline & Ecuación cuadrática & 1 & 20 & 21 & \\
\hline & $\begin{array}{l}\text { Sistema de } \\
\text { ecuaciones lineales }\end{array}$ & 1 & 21 & 26 & \\
\hline \multirow{7}{*}{ Geometría } & $\begin{array}{l}\text { Transformaciones } \\
\text { en el plano }\end{array}$ & 1 & 22 & 13 & \multirow{7}{*}{63,42} \\
\hline & \multirow{2}{*}{ Rectas y ángulos } & \multirow{2}{*}{2} & 23 & 30 & \\
\hline & & & 24 & 26 & \\
\hline & Cuerpos geométricos & 1 & 25 & 32 & \\
\hline & Semejanza & 1 & 26 & 34 & \\
\hline & Trigonometría & 1 & 27 & 35 & \\
\hline & $\begin{array}{l}\text { Relaciones de } \\
\text { proporcionalidad }\end{array}$ & 1 & 28 & 34 & \\
\hline
\end{tabular}


Continuación Tabla 1. Preguntas por subtema y número de aciertos en cada una de éstas.

\begin{tabular}{|c|c|c|c|c|c|}
\hline Temas & Subtemas & $\begin{array}{c}\text { Cantidad } \\
\text { de preguntas }\end{array}$ & $\begin{array}{c}\mathrm{N}^{\circ} \text { de } \\
\text { Pregunta }\end{array}$ & Aciertos & \% Tema \\
\hline \multirow{7}{*}{ Estadística } & \multirow{2}{*}{$\begin{array}{l}\text { Definiciones básicas } \\
\text { sobre Estadística }\end{array}$} & \multirow{2}{*}{2} & 29 & 29 & \multirow{7}{*}{47,14} \\
\hline & & & 30 & 25 & \\
\hline & \multirow{2}{*}{ Diagramas y gráficos } & \multirow{2}{*}{2} & 31 & 28 & \\
\hline & & & 32 & 20 & \\
\hline & \multirow{2}{*}{$\begin{array}{l}\text { Medidas de } \\
\text { tendencia central }\end{array}$} & \multirow{2}{*}{2} & 33 & 18 & \\
\hline & & & 34 & 20 & \\
\hline & Medidas de dispersión & 1 & 35 & 25 & \\
\hline
\end{tabular}

Fuente: Elaboración propia.

\section{Números, operaciones y figuras}

En el tema números, operaciones y figuras se observó que los estudiantes obtuvieron el menor número de aciertos en las preguntas 2 y 3 (23 y 22 respectivamente). En esos reactivos el porcentaje de aciertos está por debajo de la media aritmética que fue de 28,9 . La pregunta 2 requiere obtener la edad de una persona sabiendo que ésta es 5 años 6 meses menor y 2,8 años mayor que otras dos, mientras que en la pregunta 3 se requiere resolver un problema obteniendo $2 / 6$ de 5 . De acuerdo con lo anterior, es posible que los estudiantes tuvieran dificultades para comprender el problema de la pregunta 2, convertir 5 años 6 meses a un número decimal y al multiplicar una fracción por un número natural o entero positivo.

Al considerar que ese tipo de operaciones se requieren en varios procesos matemáticos, el dominio de éstas por parte de los estudiantes impactaría en la mejora del aprendizaje de otros contenidos matemáticos. Por ejemplo, durante los procedimientos para resolver ecuaciones se pueden presentar operaciones con números fraccionarios o decimales, incluso ambos, esto depende de la lógica de solución del estudiante.

De manera recurrente los aspirantes interpretaban de forma incorrecta la información descrita en los diferentes ejercicios; en otros casos no lograban identificar la fórmula que se debe aplicar para dar solución al problema propuesto. Un ejemplo de lo descrito anteriormente ocurre cuando al estudiante se le pide definir el volumen de una pirámide cuadrangular, y se observa que los aspirantes proponen como solución el área de un cuadrado.

\section{Magnitudes, proporciones, probabilidad y estadística}

Para evaluar el tema de magnitudes, proporciones, probabilidad y estadística que se estudió durante el curso, se incluyeron preguntas sobre los diversos subtemas y se observó que en la pregunta 8 los estudian- 
tes obtuvieron el menor número de aciertos con una frecuencia absoluta igual a 29 (Tabla 1). En esa pregunta se requiere la distancia total recorrida en kilómetros de una persona que todos los días da 5 vueltas a un parque con un perímetro que mide 3 hectómetros y 45 metros. Por ello, se observó que los estudiantes tuvieron dificultades para convertir de un múltiplo de una unidad de medida a otro múltiplo, o bien, de un múltiplo a la unidad de medida, o de la unidad de medida a un múltiplo de la magnitud longitud.

Los aspirantes también presentaron dificultades para convertir una unidad de medida del sistema anglosajón a una del sistema internacional o viceversa; además se identificaron dificultades para resolver problemas sobre reparto proporcional, o bien, para obtener el valor unitario en una situación de proporcionalidad directa. Otras dificultades que se identificaron fueron al resolver problemas de tipo valor faltante y calcular la variable dependiente o independiente en una relación de correspondencia asociada con una situación de proporcionalidad directa.

Además, los estudiantes tuvieron algunas problemáticas para obtener una fracción equivalente, relacionar el porcentaje con su representación fraccionaria, y en consecuencia, al comparar una fracción con un porcentaje para determinar cuál es mayor.

Un caso particular del tema de magnitudes, proporciones, probabilidad y estadística se presenta en la pregunta 14, cuyo cuestionamiento pide al estudiante definir el valor de la calificación faltante para definir el promedio que se conoce, lo que evidencia las debilidades de los estudiantes al plantear y resolver una ecuación como la siguiente:

\section{$8.5=\frac{7+b+9}{3}$}

\section{Álgebra}

Para el tema de álgebra, se observó que las preguntas en las que los estudiantes obtuvieron el menor número de respuestas correctas fueron la 15 y 20 con 23 y 21 aciertos respectivamente (Tabla 1). En la pregunta 15 se pedía identificar cuál de las siguientes fracciones: $9 / 10,7 / 10,8 / 10$ o $5 / 6$, era mayor que $7 / 8$, lo que implica convertir cada fracción a su representación decimal y comparar números decimales, o bien, convertir las fracciones de manera que fueran equivalentes entre sí y comparar sus numeradores. La pregunta 20 se asocia con la resolución de una ecuación cuadrática incompleta $5 x^{2}-2 x=x^{2}+6 x$, cuya solución implica reducir términos semejantes, igualar la expresión a 0 , factorizar la expresión resultante y resolver la ecuación lineal que se deriva de cada factor para obtener el valor de $\mathrm{x}^{1} \mathrm{y} \mathrm{x}^{2}$.

Lo anterior, pone de manifiesto que los aspirantes a la EBUAQ tuvieron dificultades al comparar números fraccionarios con diferente denominador y resolver ecuaciones cuadráticas incompletas por medio de la factorización.

Otras dificultades como convertir números fraccionarios a decimales o viceversa, además de sus limitaciones para definir fracciones equivalentes y ordenar cantidades decimales o fraccionarias, fueron evidentes en las respuestas dadas por los estudiantes. 
En la pregunta 17 se le solicita definir al estudiante el quinto término de una sucesión con progresión aritmética iniciando con una cantidad negativa y sumando una positiva; algunos estudiantes proponen como solución un número negativo de mayor magnitud que el inicial, lo que evidencia las dificultades que tienen los aspirantes al sumar cantidades con signos diferentes.

Igualmente, se identificó que los aspirantes cometen errores al aplicar las leyes de los exponentes, lo que se aprecia en la pregunta 18 que propone una suma de polinomios y cuyo resultado por parte de los estudiantes describe que al agrupar términos semejantes los exponentes de las bases deben sumarse. Otro conflicto de los estudiantes fue lograr comprender la relación de los pares ordenados con una función lineal, suponiendo que si un par, de un listado de pares ordenados, satisface a la función lineal, se afirma que todos los demás también.

También, se observaron problemáticas para dar solución a ecuaciones cuadráticas con una sola incógnita cometiendo errores de factorización, al despejar y al reducir términos semejantes. Además, los estudiantes tienen dificultades al resolver sistemas de ecuaciones de 2x2, logrando plantear el método de solución de manera correcta, pero errando durante el desarrollo del procedimiento.

De manera general, en los contenidos de álgebra además del dominio teórico y metódico, se requiere de una capacidad de interpretación analítica para definir de qué manera aplicar cada uno de los procesos. Durante el estudio de esta rama de las matemáticas, el estudiante toma ciertas decisiones que deben estar justificadas en la teoría y lógica matemática, lo que en ocasiones los lleva al error.

\section{Geometría}

Al revisar el tema de geometría se incluyeron los diversos subtemas y se observó que en la pregunta 23 los estudiantes obtuvieron el menor número de aciertos (Tabla 1). En esa pregunta se muestran dos ángulos suplementarios, la medida de uno de los ángulos se representa por medio de una expresión algebraica, mientras que la del otro es igual a $45^{\circ}$ y se requiere determinar el valor de la incógnita (Figura 2). La solución de este reactivo implica plantear una ecuación de primer grado y resolverla, por lo que los estudiantes tuvieron dificultades al respecto. Este resultado coincide con el bajo porcentaje de respuestas correctas en preguntas sobre álgebra (Figura 1).

Tabla 2. Pregunta 23 de Geometría como parte del subtema rectas y ángulos.

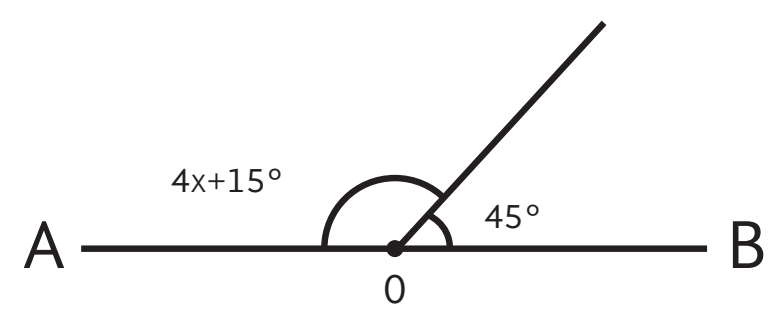

Fuente: Elaboración propia.

En el reactivo 22 los estudiantes tuvieron dificultades en la comprensión del problema puesto que es posible que interpretaran que el punto A está a una distancia de $6 \mathrm{~cm}$ del punto en el que éste se refleja. Algo que también fue evidente es que los estudiantes presentan deficiencias para identificar los ángulos formados entre rectas paralelas cortados por una secante, además de confundir sus propiedades; tal es el caso del reactivo 23 donde suponen que los ángulos adyacentes son congruentes. 
Otro reactivo que presentó conflictos fue el 25, donde se propone al estudiante comparar la magnitud volumétrica de dos prismas, lo que mostró dificultades para definir su volumen y para dividir números con varios ceros. En el reactivo 26 los estudiantes confunden el seno de M ( $2 / 7)$ con el coseno de M $(\sqrt{3} / 7)$, así que se propone un proceso de solución incorrecto.

\section{Estadística}

Finalmente, en el tema de estadística se evaluó desde definiciones básicas hasta medidas de disper- sión. En la Tabla 1 se puede ver que en el reactivo 33 los estudiantes obtuvieron la menor cantidad de respuestas correctas (18 aciertos). En éste se requería el cálculo de las medidas de dispersión (moda, media y mediana) a partir de la información contenida en un histograma de frecuencias (Figura 3). Los aspirantes obtuvieron el porcentaje más bajo de aciertos en esta pregunta a pesar de haber estudiado al respecto en el curso propedéutico, tal vez porque el contenido matemático asociado a ésta se estudia hasta el cuarto bloque de tercer año de secundaria, es decir, después de haber presentado el examen del curso propedéutico.

Tabla 3. Preguntas 33 y 34 de estadística como parte del subtema medidas de tendencia central.

33. El siguiente grafico representa las calificaciones obtenidas de un grupo de estudiantes de bachillerato en la clase de Química I. Determinar el valor de su media, mediana y moda.

\section{Frecuencia}

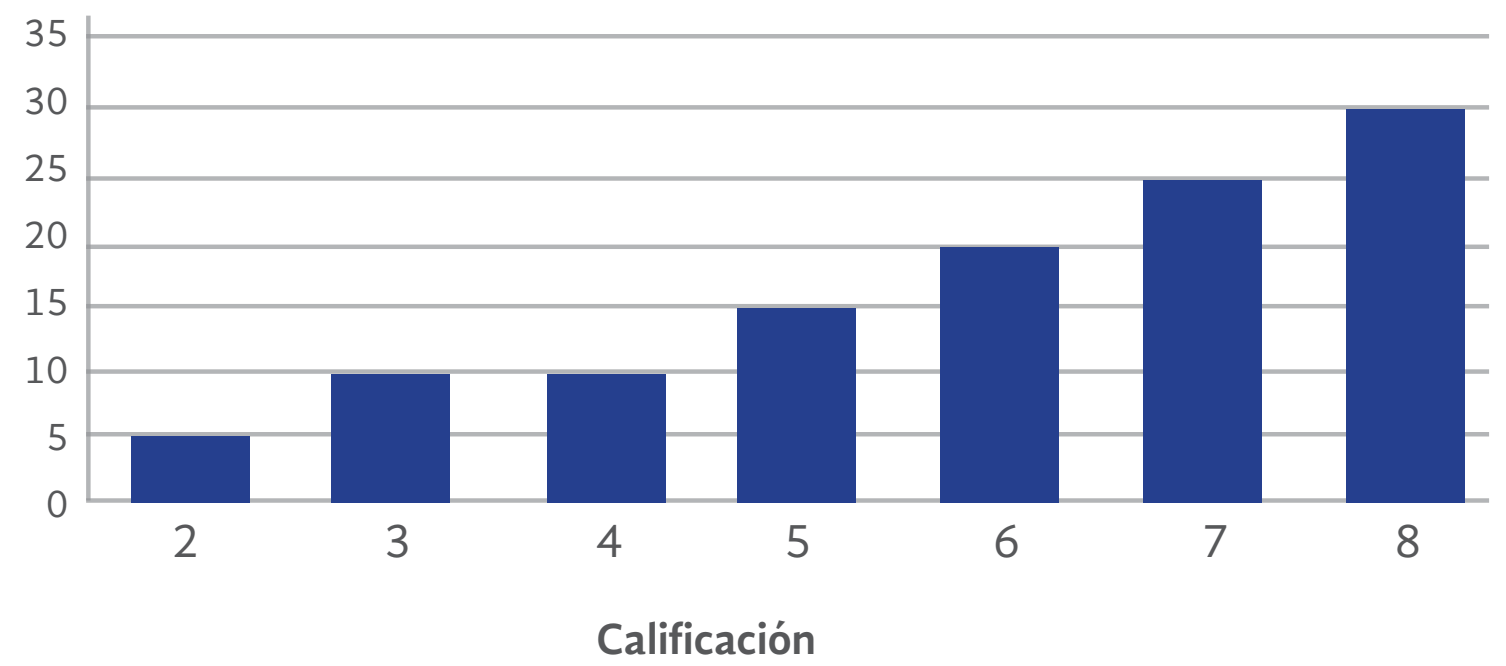

34. Un camión realizó 14 recorridos, transportando en cada viaje a 13, 14, 15, 9, 5, 2, 14, 10, 6, 10, 11, 13, 14,14 pasajeros respectivamente. Determina la media, moda y mediana de estos datos.

Fuente: Elaboración propia. 
Aunque también existe la posibilidad de que las dificultades se deban a la instrucción sobre el cálculo de medidas de tendencia central, ya que en la mayoría de los casos se proponen actividades en las que se obtengan esas medidas partiendo de un conjunto de datos, en lugar de hacerlo a partir de un histograma de frecuencias absolutas.

Es importante aclarar en el reactivo 33, como en el 34, se pidió a los aspirantes de la EBUAQ calcular la media, mediana y moda; en este último, obtuvieron la mayor cantidad de respuestas correctas. Como se puede ver en la Figura 3, la diferencia entre ambos reactivos radica en la forma en la que se presentan los datos; en el 33 por medio de un histograma de frecuencias y en el 34 como un conjunto de datos, es decir, en la primera pregunta el aspirante debía analizar e interpretar la gráfica para obtener los datos, mientras que en la segunda sólo tenía que ordenarlos.

Las primeras preguntas del tema de estadística evaluaron el dominio conceptual por parte de los aspirantes y los resultados obtenidos evidenciaron que algunos aspirantes no tienen una conceptualización correcta de los términos estadísticos. Por ejemplo, sus respuestas mostraron que no saben distinguir entre la estadística descriptiva e inferencial, entre un dato y una variable, y así mismo, les falta claridad sobre lo que caracteriza a cada uno de los distintos gráficos que existen.

\section{Discusión y reflexiones}

El porcentaje de respuestas correctas que obtuvieron los 50 estudiantes que tomaron el curso propedéutico (grupo experimental) fue del $57,88 \%$, lo que constituye una mejora ya que en 2018 la media de aciertos en la prueba EXCOBA fue del 42,26 \% y en 2019 del 47,39\%. Se observa entonces una diferencia de 15,62 y 10,49 puntos porcentuales respectivamente con respecto a los dos últimos años.

Al evaluar el examen de matemáticas propuesto al finalizar el curso propedéutico, se identificó una diferencia significativa entre los resultados obtenidos por los estudiantes que tomaron el curso propedéutico -grupo experimental-, en comparación con los que no lo tomaron -grupo de control-.

Se aprecia en el examen de matemáticas del curso propedéutico (Figura 1) que el mayor porcentaje obtenido por los estudiantes se vincula con preguntas sobre magnitudes, proporciones, probabilidad y estadística $(68,28 \%)$, mientras que el menor con reactivos sobre estadística $(47,14 \%)$.

De manera general, los estudiantes tuvieron mayores dificultades para interpretar datos incluidos en una gráfica, que los datos cuando hacen parte de una situación problema. Aun así, una cantidad considerable de estudiantes no comprenden de forma apropiada los textos que describen cada una de las problemáticas propuestas. Los procesos cuya metodología exigen un análisis y toma de decisiones presentan mayor complejidad a los estudiantes, que aquellos cuyo proceso de solución consiste en recrear métodos definidos.

De esta forma, tener un conocimiento sólido de los temas de estudio que anteceden y se involucran en el actual curso, disminuye los errores cometidos por el estudiante durante sus procesos de solución.

Es importante remarcar que la experiencia de trabajar en un entorno virtual es más demandante, debido a que requiere de una automotivación para revisar los contenidos y desarrollar las actividades 
propuestas. Por esta razón, el desarrollo se enfocó en los contenidos desde esta perspectiva, tomando en cuenta que el estudiante no tendría un acompañamiento sincrónico durante sus sesiones de estudio, y que además cuenta con una carga de actividades virtuales. Reflexionar sobre las situaciones que actualmente atraviesan los estudiantes es vital a la hora del desarrollo e implementación de cualquier curso, puesto que su contexto ha cambiado radicalmente y esto afecta la forma en que aprende.

En estas nuevas condiciones que los estudiantes afrontan, se debe entender que sin importar la estrategia didáctica que se implemente con el apoyo de las TIC para propiciar un aprendizaje significativo en los estudiantes, es necesario que se comprometan y responsabilicen en la gestión de su proceso de estudio.ed 


\section{Referencias}

Cabero, J. (2015). Nuevos retos en tecnología educativa. (4ta ed.). Editorial Síntesis.

Caletti, Y. y Romero, E. (2015). La utilidad de los Entornos Virtuales de Enseñanza y Aprendizaje. (EVE-A) en relación al proceso educativo. Experiencias De Aprendizaje Mediadas Por Las Tecnologias Digitales. Pautas Para Docentes Y Diseñadores Educativos. UNAM.

Cuevas, C. (2014). Es grave en México el problema de reprobación en matemáticas, advierte investigador. Vanguardia. https://vanguardia.com.mx/ esgraveenmexicoelproblemadereprobacionenmatematicasadvierteinvestigador-2155718.html

Hernández, R., Fernández, C. y Baptista L. (2010). Definiciones de los enfoques cuantitativo y cualitativo, sus similitudes y diferencias. Metodología de la investigación (5ta. Ed.). McGraw Hill.

Organización para la Cooperación y el Desarrollo Económicos. (2018). Resultados PISA 2015. https://www.oecd.org/pisa/39730818.pdf
Secretaría de Educación Pública. (2015). Planea. Resultados Nacionales 2015. Educación Media Superior: Lenguaje y comunicación Matemáticas. http://www.seslp.gob.mx/consejostecnicosescolares/PRIMARIA/4-3aSesionOrdinaria/RESULTADOSPLANEA.pdf

Secretaría de Educación Pública. (2017). Planea. Resultados Nacionales 2017. Educación Media Superior: Lenguaje y comunicación Matemáticas. http:// planea.sep.gob.mx/content/general/docs/2017/ ResultadosNacionalesPlaneaMS2017.PDF

Secretaría de Educación Pública. (2018). Planea. Resultados Nacionales 2018. Educación Media Superior: Lenguaje y comunicación Matemáticas. https://historico.mejoredu.gob.mx/evaluaciones/ planea/resultados-planea/

Secretaría de Salud. (2020). Presentan Salud $y$ SEP medidas de prevención para el sector educativo nacional por COVID-19. https://www.gob. $\mathrm{mx} /$ salud/prensa/presentan-salud-y-sep-medidas-de-prevencion-para-el-sector-educativo-nacional-por-covid-19 